Supplement of Solid Earth, 10, 27-57, 2019

https://doi.org/10.5194/se-10-27-2019-supplement

(C) Author(s) 2019. This work is distributed under

the Creative Commons Attribution 4.0 License.

(c) (i)

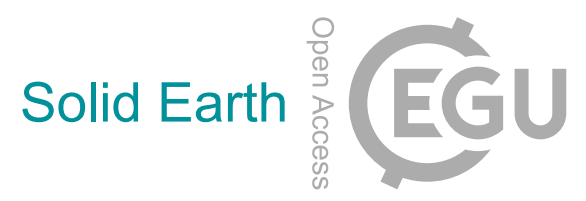

Supplement of

\title{
A semi-automated algorithm to quantify scarp morphology (SPARTA): application to normal faults in southern Malawi
}

Michael Hodge et al.

Correspondence to: Michael Hodge (hodgems@cardiff.ac.uk)

The copyright of individual parts of the supplement might differ from the CC BY 4.0 License. 
Introduction

In the manuscript, we presented a misfit plot between the scarp height calculated by the algorithm compared to a manual analysis, for the synthetic catalogues (Fig. 4 and 5). Here, we present the results for scarp width and slope for the noise free synthetical scarp catalogue (Figs. S1 and S2; Table S1) and the noisy synthetic catalogue (Figs. S3 to S4; Table S2). All plots are for selected profiles, as the catalogues comprised over 1,000 profiles. For a description as to why these profiles were chosen, please refer to the relevant sections of the manuscript (i.e., sections 3.2 and 3.3).

One of the research questions in the manuscript was to what degree does DEM resolution influence the performance of the algorithm and/or the interpretation of structural segments. Here, we provide the results of a resolution analysis performed on the synthetic scarp catalogues. Figs. S5 to S7 show the average misfit for all 1,000 noisy catalogue scarps for resolutions of 5 , 10 and $30 \mathrm{~m}$, for three selected filter types (Savitzky-Golay, Median and Lowess). A greater number of scarps were identified using the higher resolution data, especially those with gentle slopes. In addition, the misfit generally reduced with increasing resolution.

In addition, Figs. S8 and S9 (and Tables S3 and S4) show the misfit analysis for 12 and $30 \mathrm{~m}$ resolution profiles for the BMF, compared to the $5 \mathrm{~m}$ resolution used in manuscript to infer structural segments along the fault. A tabular version of the manual analysis is shown in Table S3. The maximum scarp height, width and slope values calculated by the manual analysis is shown in Table S6. Similar to the synthetic results above, the higher the DEM resolution, the greater than number of scarps identified (Table S5). A comparison between estimated scarp height, width and slope for each DEM resolution is shown in Fig. S10. The bottom panel (b) shows the results following the removal of outliers (for tabular form, see Table S7).

The along-strike difference between DEM resolutions for the BMF is shown in Fig. S11 following the removal of outliers (as described in the Appendix of the manuscript). It shows that the scarp height estimate is least influenced by the DEM resolution, and scarp width the most affected. A more detailed comparison for each scarp parameter, and the along-strike variation in scarp height is shown in Fig. S12.

Lastly, Figs. S13 to S17 show algorithms results against a manual analysis for: the Thyolo fault (Fig. S13), the Muona fault (Fig. S14), and the Malombe fault scarps (Figs. S15 to S17). The DEM used was a $12 \mathrm{~m}$ TanDEM-X DEM. The bottom plot shows the along-strike variation in scarp height, width and slope estimated by a manual analysis compared to the algorithm. The results for the algorithm are based on the best-fit parameter space for each fault. The best-fit parameter space can be found in the manuscript. 


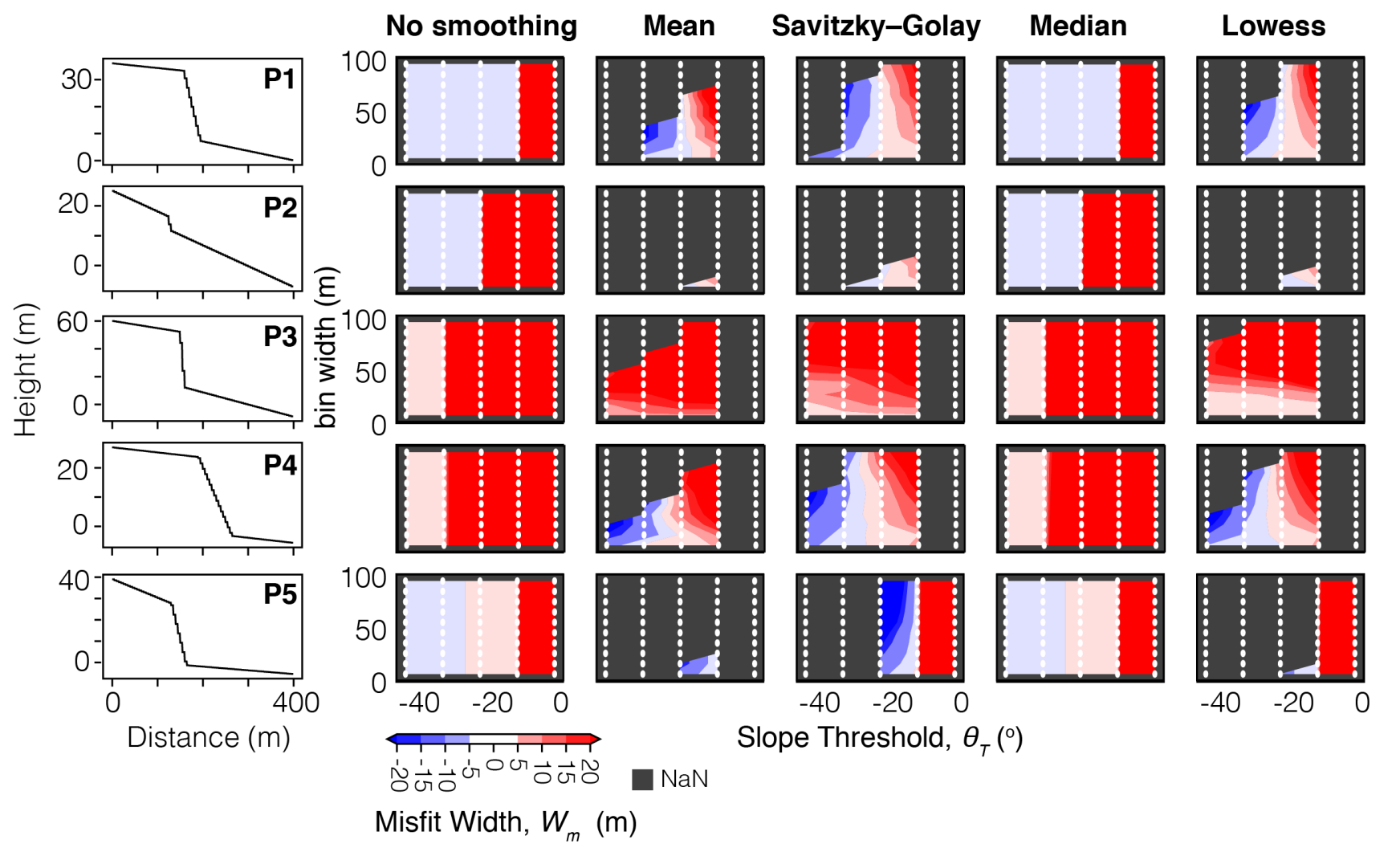

Figure S1. Scarp width misfit $\mathrm{W}_{m}$ for five noise-free synthetic catalogue examples (see Table S1): 1) randomly selected; 2) small scarp height; 3) steep, large scarp; 4) gentle original surfaces; and 5) variable original surfaces. See Fig. 4 for scarp heighth misfit results and Fig. $\mathrm{S} 2$ for scarp slope $\mathrm{a}_{\mathrm{m}}$ misfit results. 


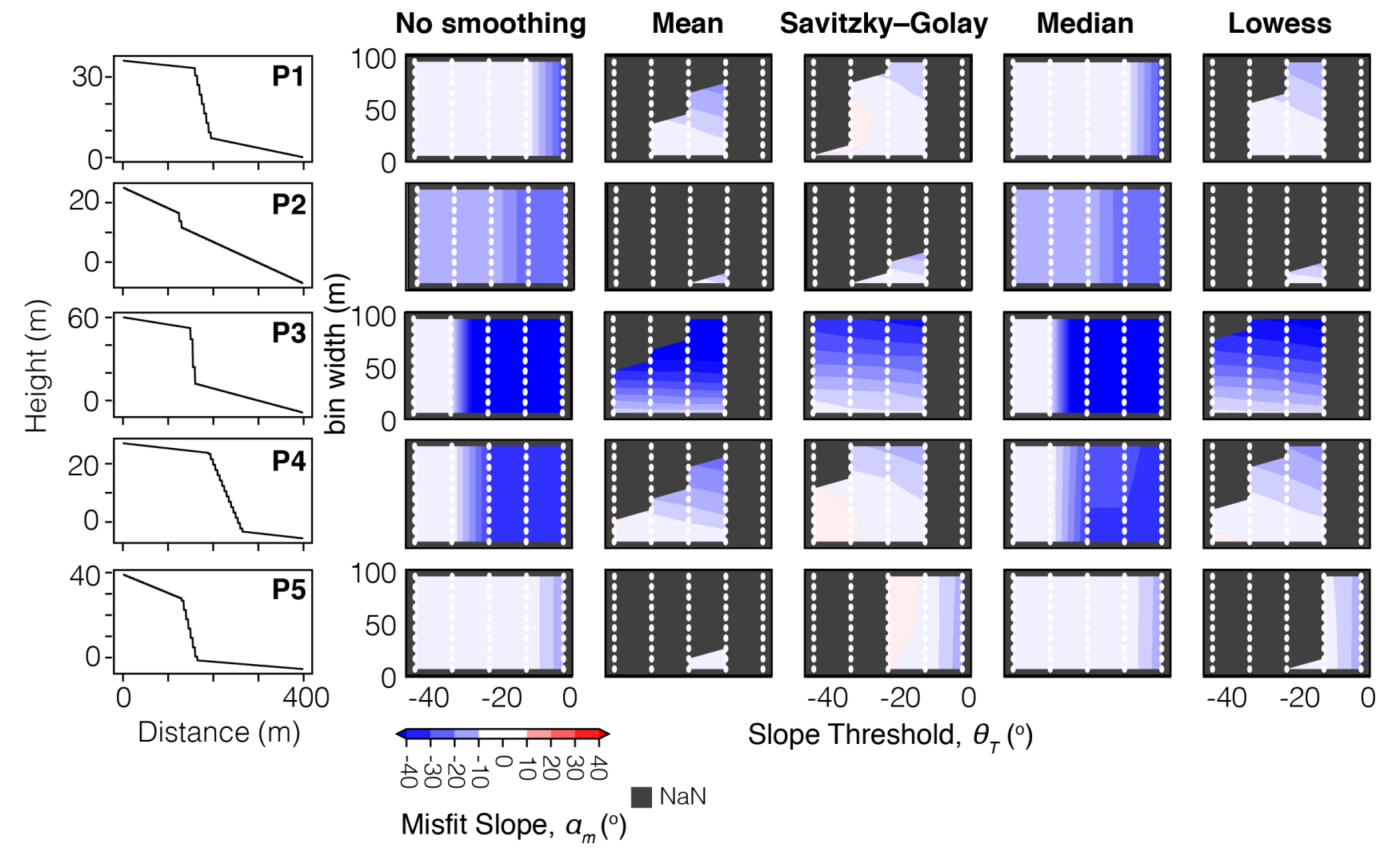

Figure S2. Scarp slope misfit $W_{m}$ for five noise-free synthetic catalogue examples (see Table S1): 1) randomly selected; 2) small scarp height; 3) steep, large scarp; 4) gentle original surfaces; and 5) variable original surfaces. See Fig. 4 for scarp height $H_{m}$ misfit results and Fig. S1 for scarp width $W_{m}$ misfit results. 


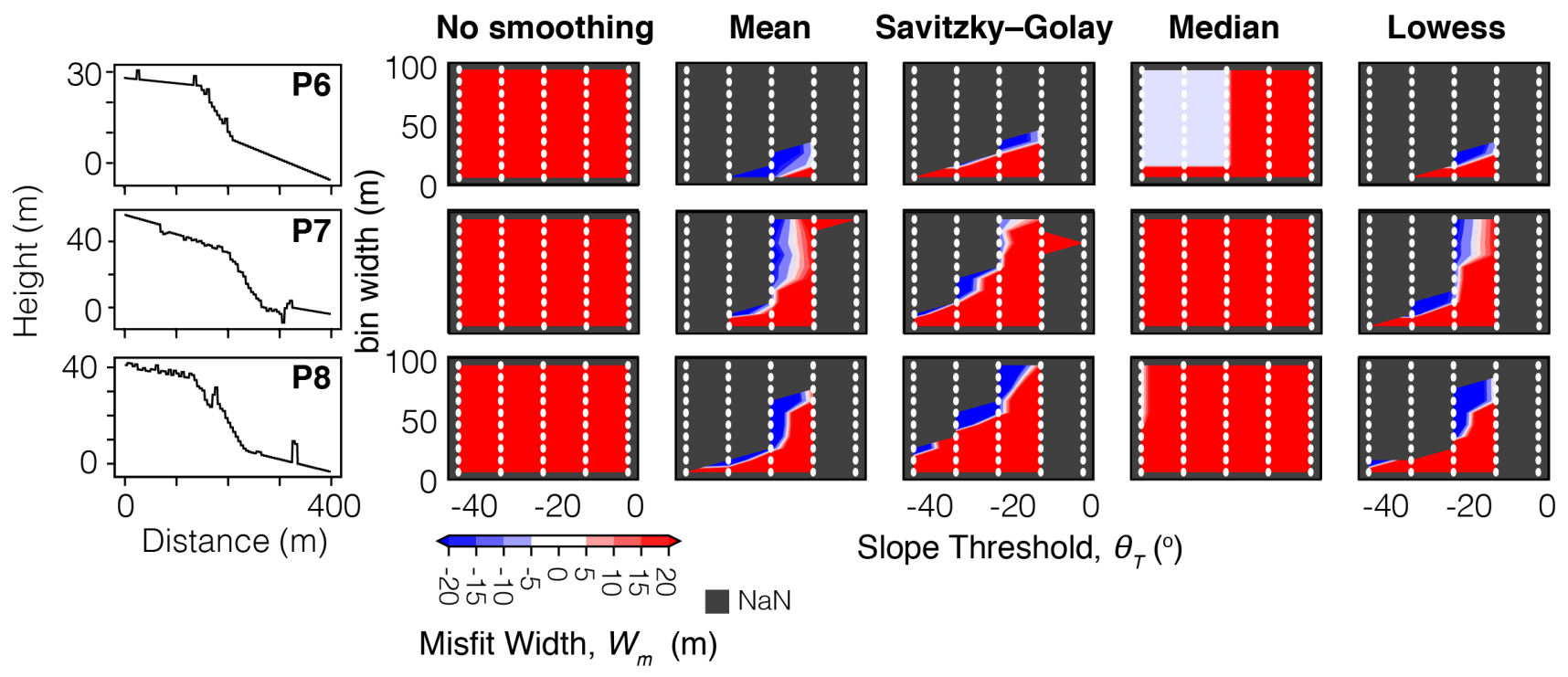

Figure S3. Scarp width misfit $W_{m}$ for three noisy synthetic catalogue examples (see Table S2): 6) high signal-to-noise; 7) medium signalto-noise; and 8) low signal-to-noise. See Fig. 4 for scarp height $H_{m}$ misfit results and Fig. S4 for scarp slope $\alpha_{m}$ misfit results.

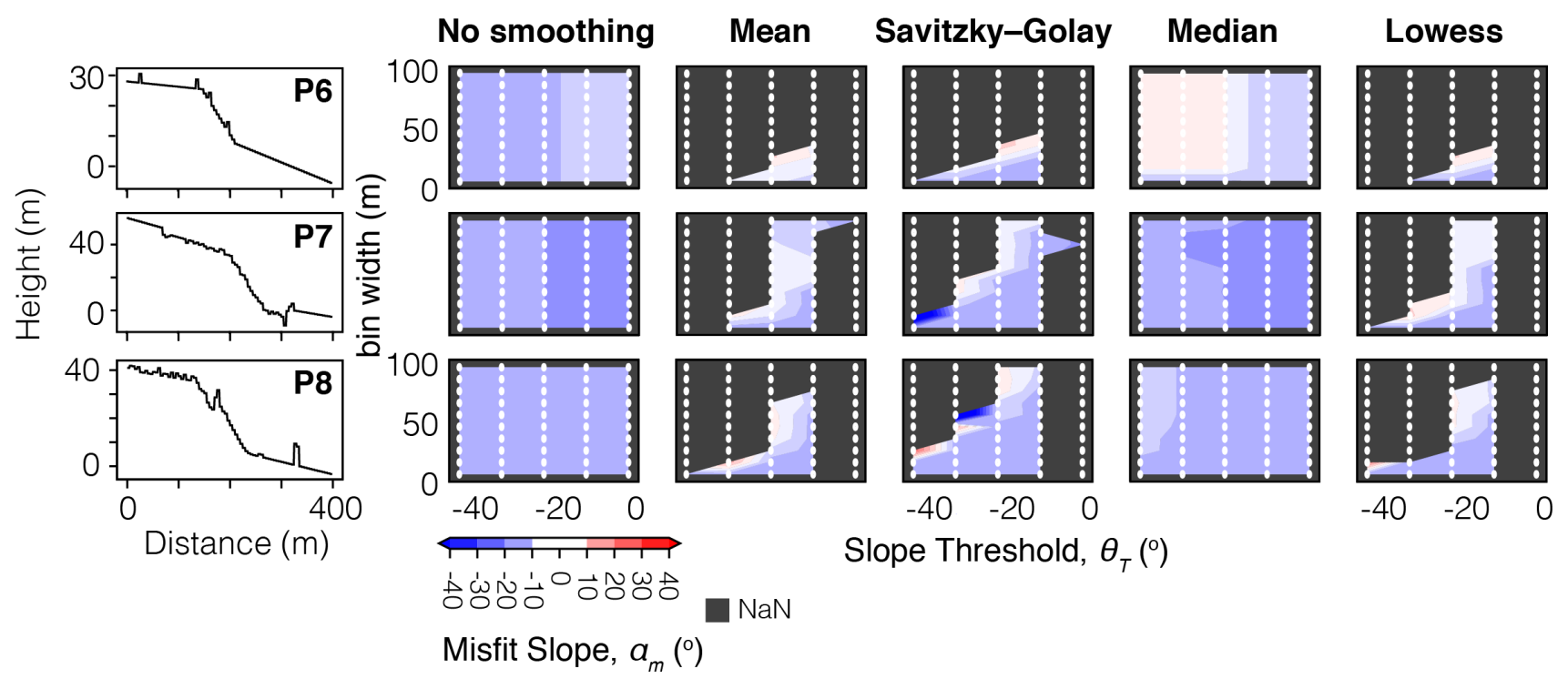

Figure S4. Scarp slope misfit $\alpha_{m}$ for three noisy synthetic catalogue examples (see Table S2): 6) high signal-to-noise; 7) medium signal-tonoise; and 8) low signal-to-noise. See Fig. 4 for scarp height $H_{m}$ misfit results and Fig. S3 for scarp width $W_{m}$ misfit results. 


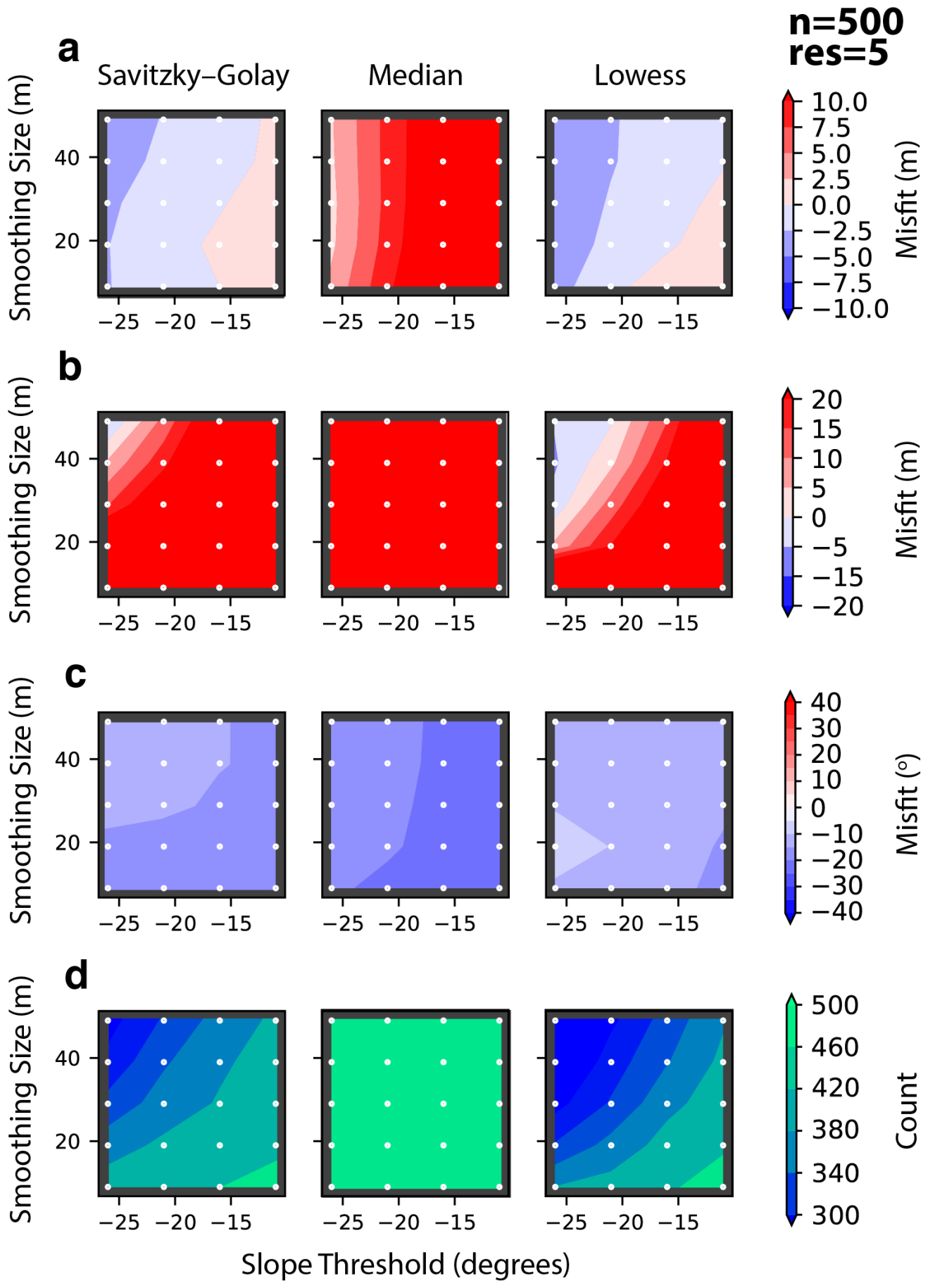

Figure S5. The average misfit and count for all 1,000 noisy synthetic catalogue fault scarps using a resolution of $5 \mathrm{~m}$ : a) scarp height; b) scarp width; c) scarp slope; and d) count. Grey values denote a NaN result was returned for all profiles. 


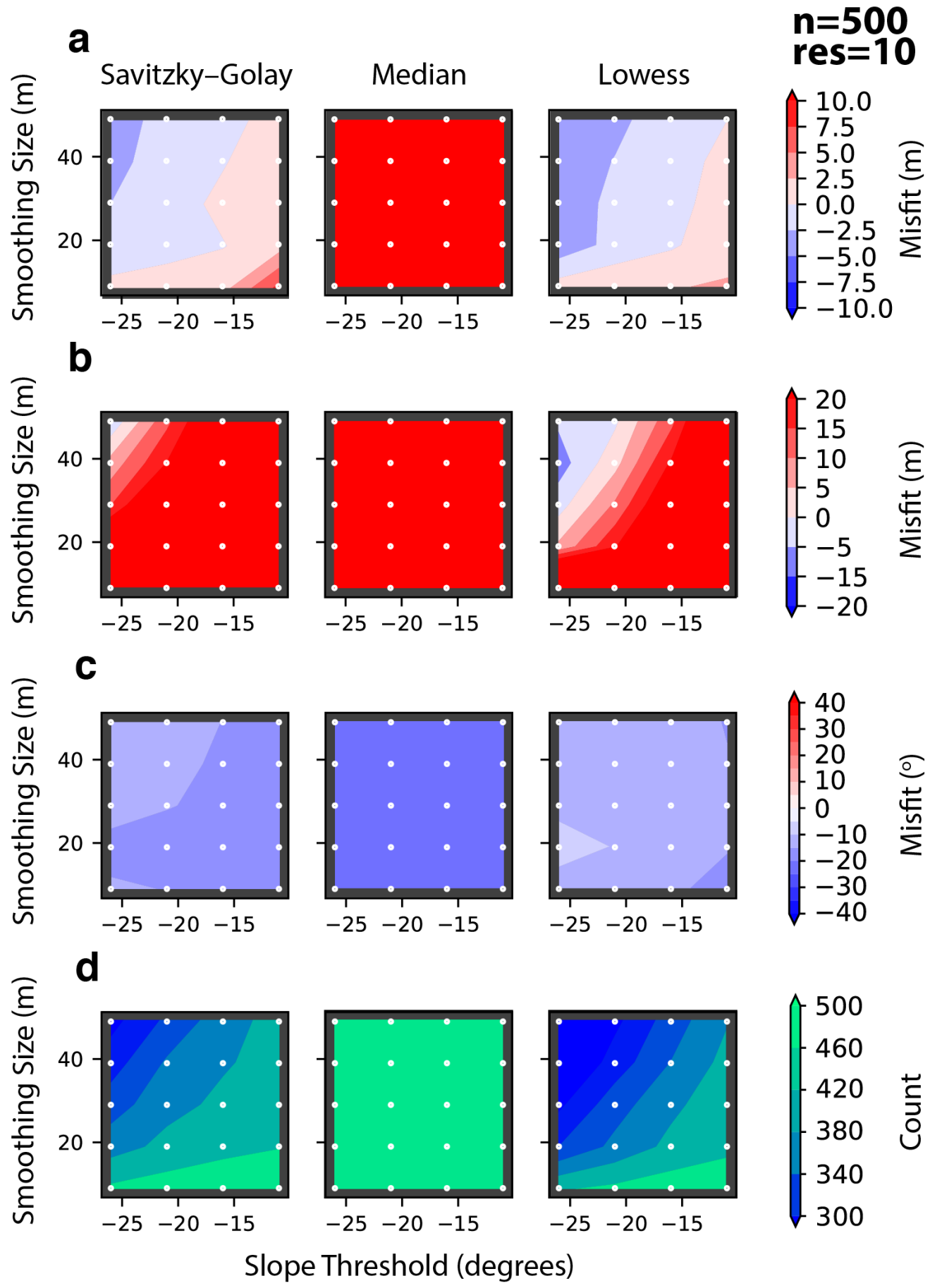

Figure S6. The average misfit and count for all 1,000 noisy synthetic catalogue fault scarps using a resolution of $10 \mathrm{~m}$ : a) scarp height; b) scarp width; c) scarp slope; and d) count. Grey values denote a NaN result was returned for all profiles. 

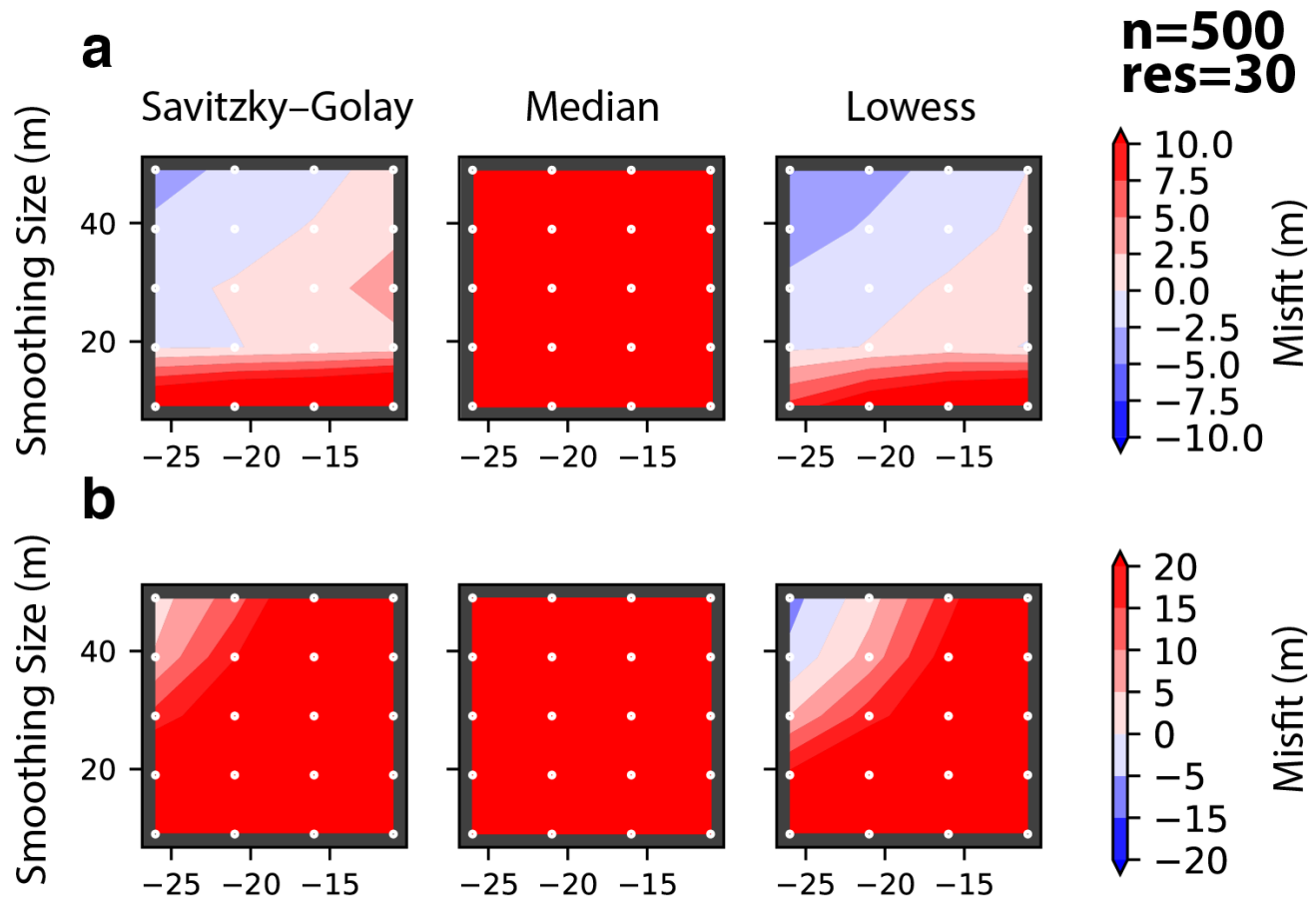

\section{C}
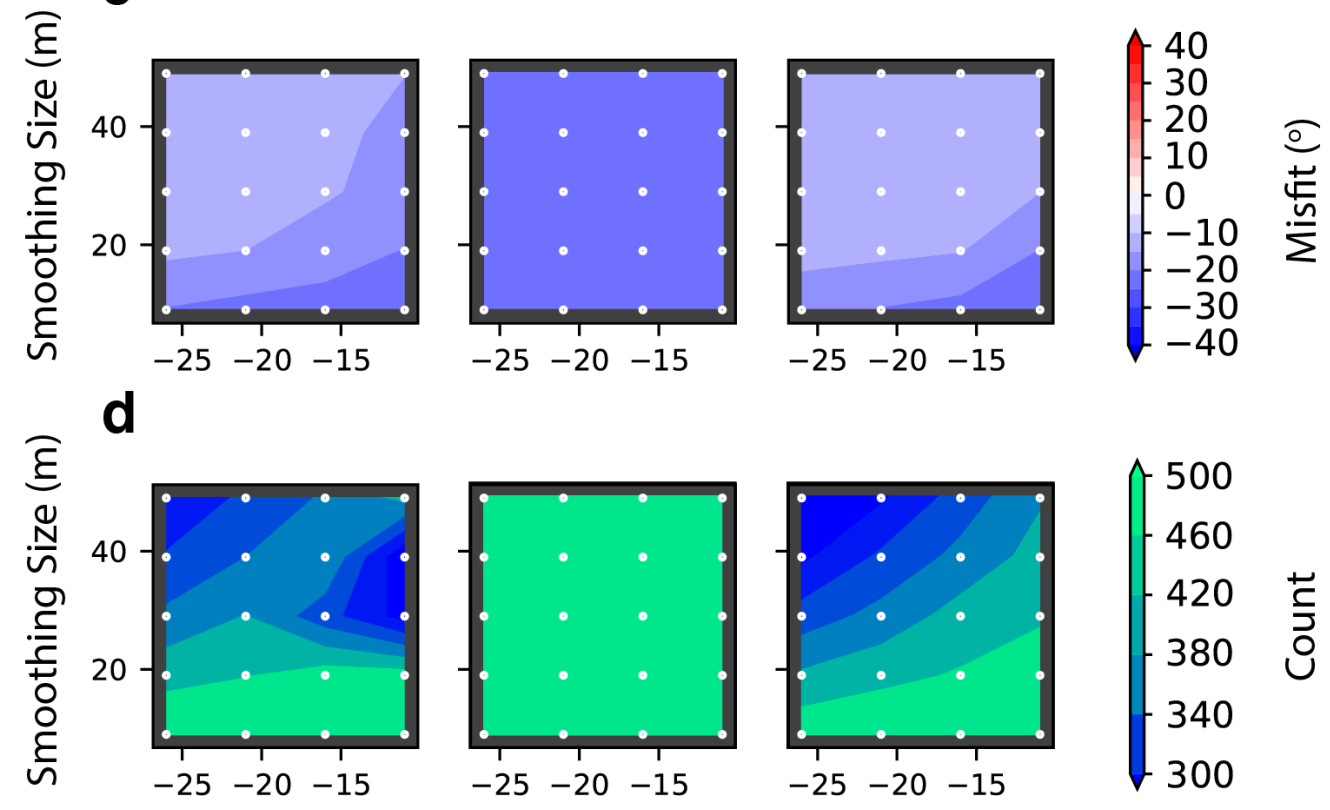

Slope Threshold (degrees)

Figure S7. The average misfit and count for all 1,000 noisy synthetic catalogue fault scarps using a resolution of 30 m: a) scarp height; b) scarp width; c) scarp slope; and d) count. Grey values denote a NaN result was returned for all profiles. 

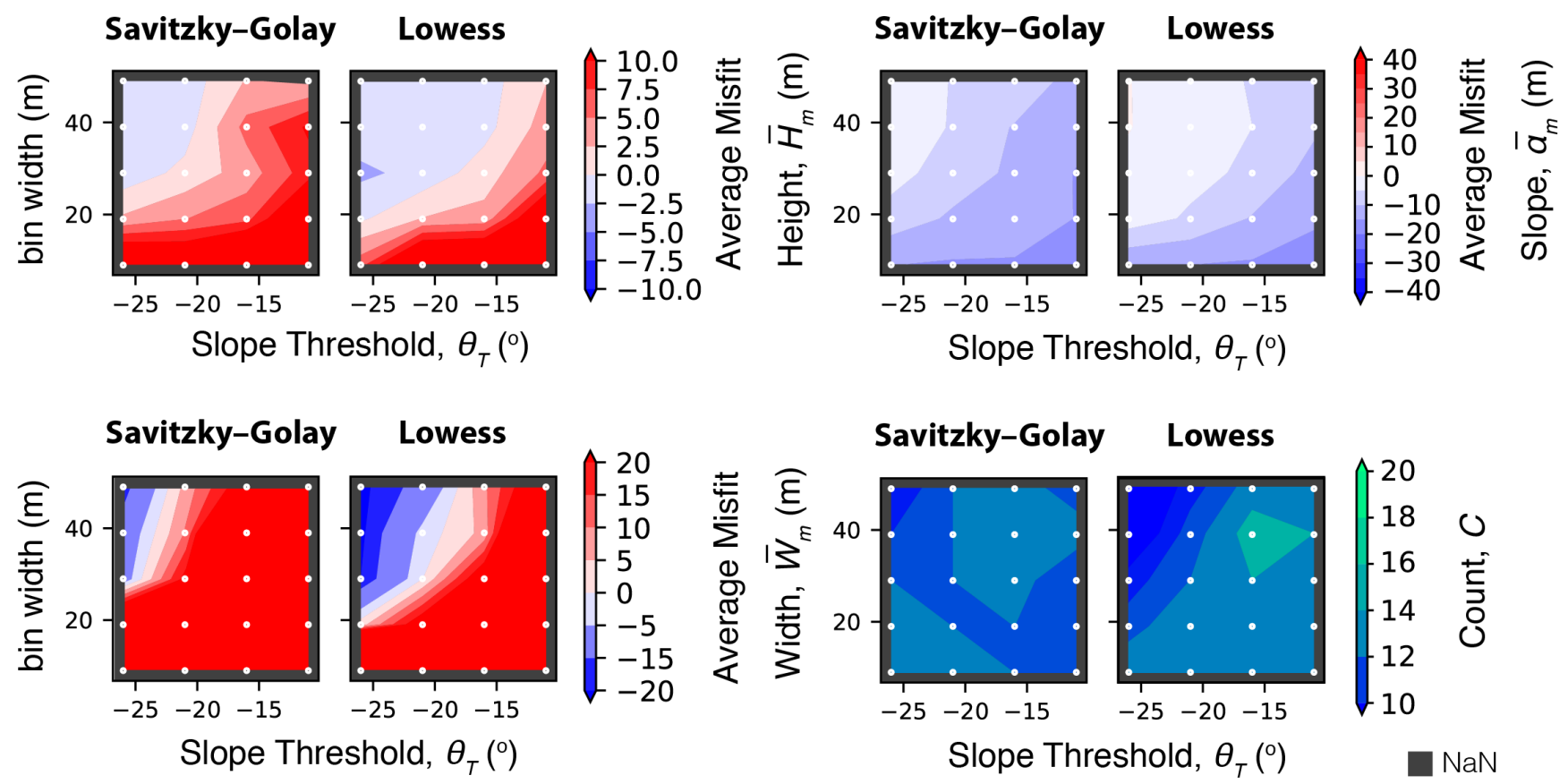

Figure S8. Average misfit values between algorithm and manual scarp parameters using the TanDEM-X $12 \mathrm{~m}$ DEM for: a) scarp height $\bar{H}_{m}$; b) scarp width $\bar{W}_{m}$; and c) scarp slope $\bar{\alpha}_{m}$. d) The count $(C)$ of identified fault scarps. 

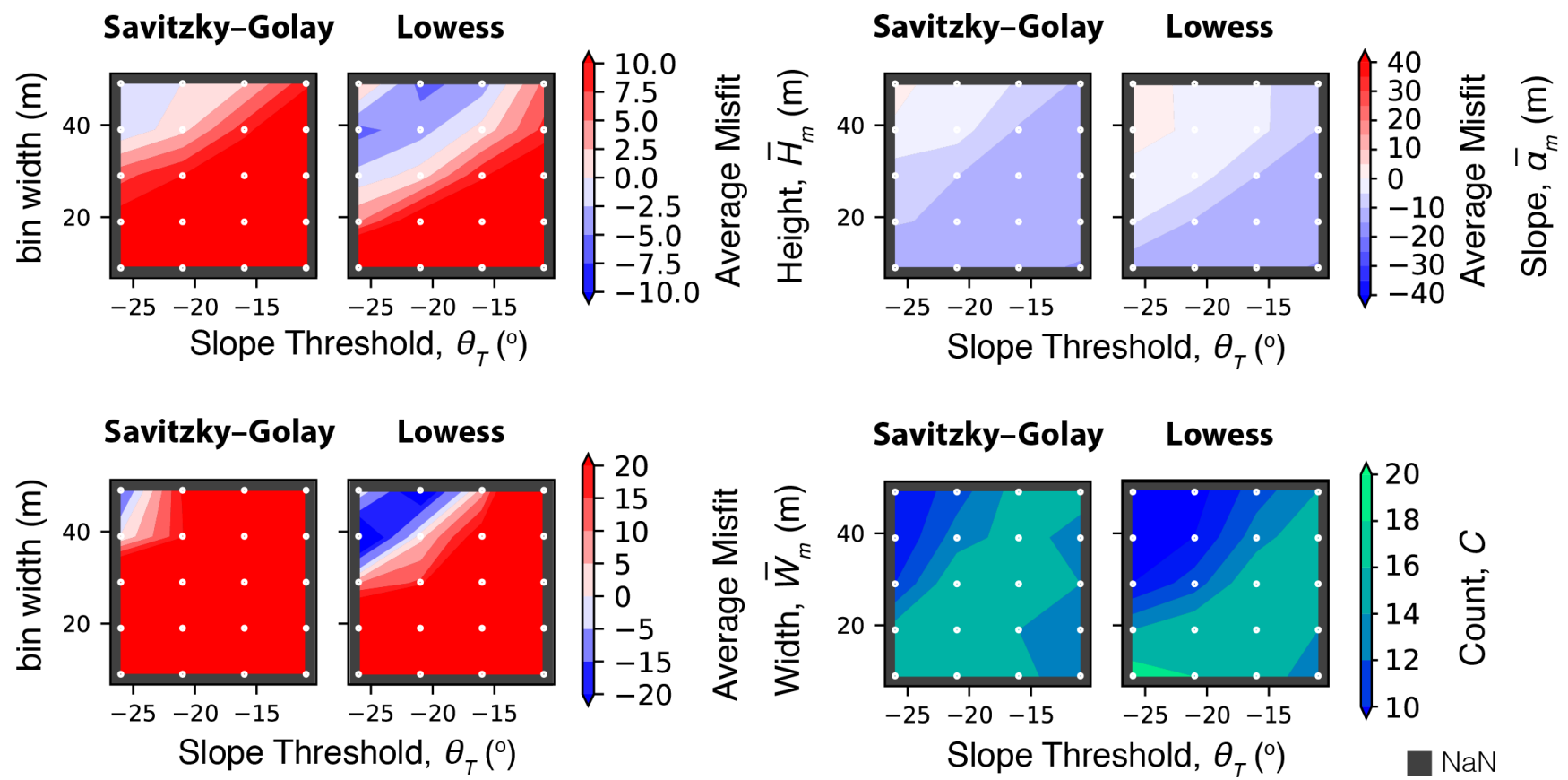

Figure S9. Average misfit values between algorithm and manual scarp parameters using the SRTM $30 \mathrm{~m}$ DEM for: a) scarp height $\bar{H}_{m}$; b) scarp width $\bar{W}_{m}$; and c) scarp slope $\bar{\alpha}_{m}$. d) The count $(C)$ of identified fault scarps. 

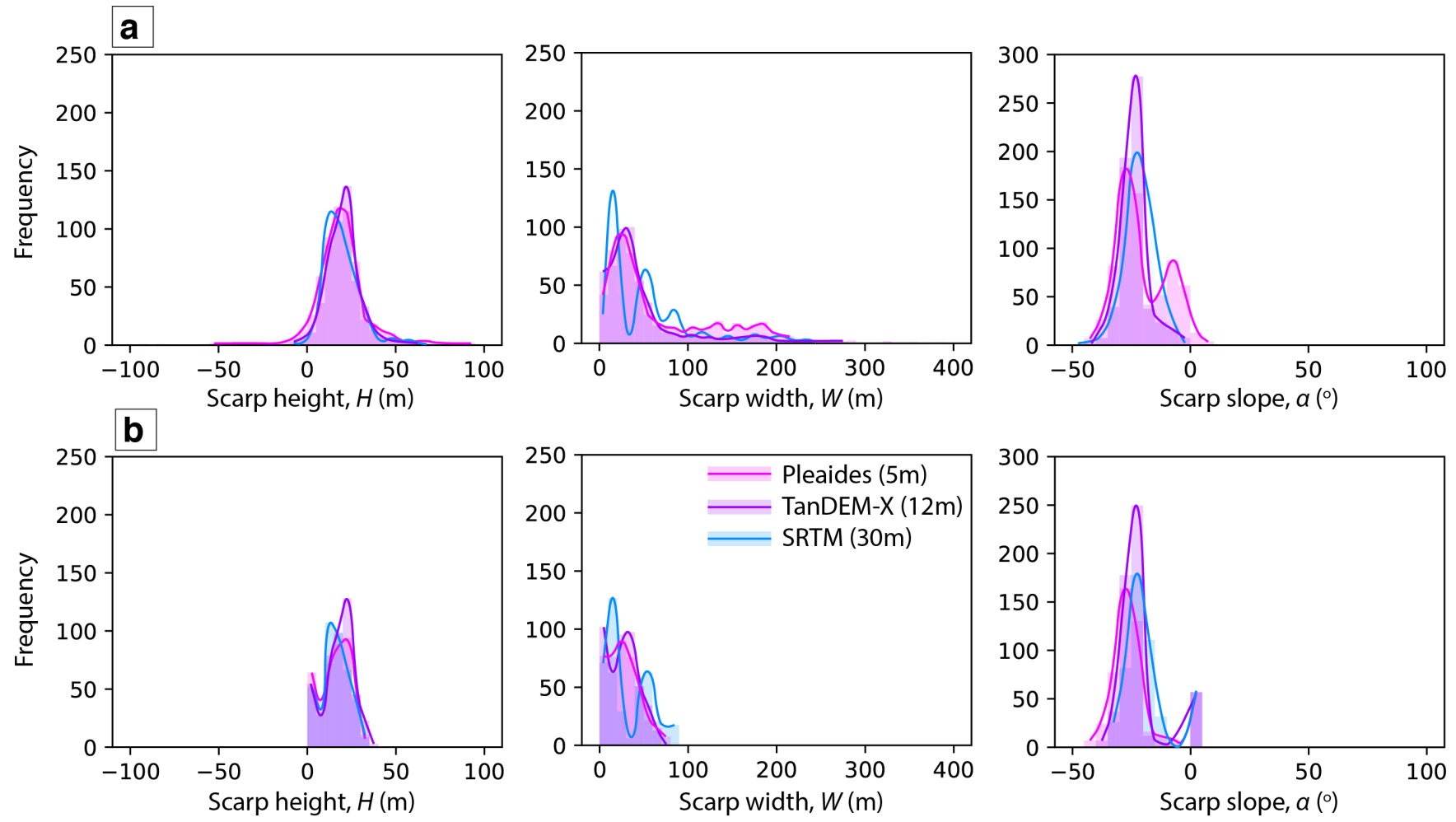

Figure S10. Histogram of the Bilila-Mtakataka fault scarp parameters using the algorithm for: a) all algorithm estimates (raw); and b) post-quality checked results. Pleiades $5 \mathrm{~m}$ (pink), TanDEM-X $12 \mathrm{~m}$ (purple) and SRTM $30 \mathrm{~m}$ (blue). 

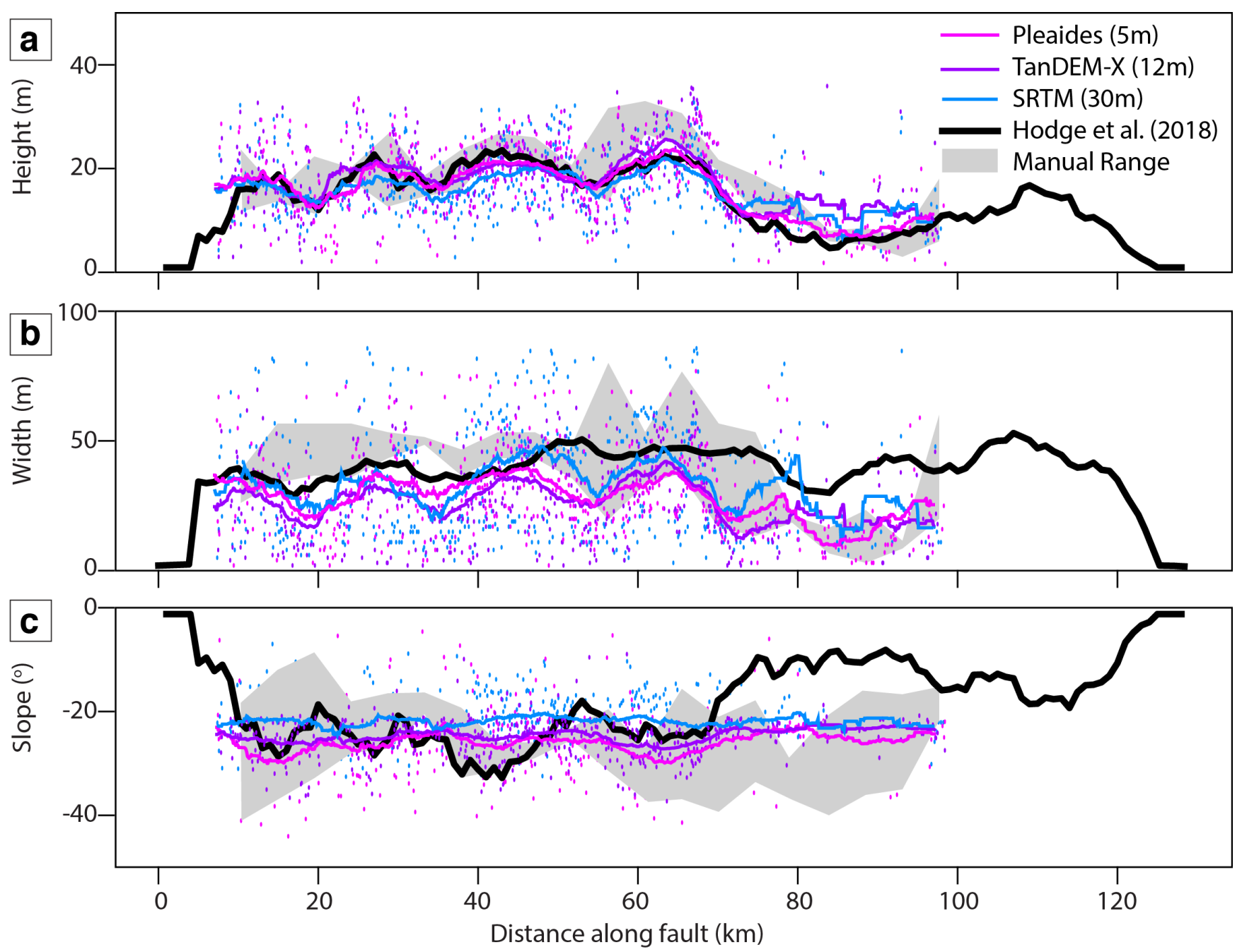

Figure S11. The quality checked height, width and slope profiles using the algorithm on Pleiades $5 \mathrm{~m}$ (pink), TanDEM-X $12 \mathrm{~m}$ (purple) and SRTM $30 \mathrm{~m}$ (blue) DEMs. A moving mean (window size of $7 \mathrm{~km}$ ) is shown by a solid line coloured corresponding to the DEM used. The manually derived TanDEM-X moving mean (window size of $7 \mathrm{~km}$ ) from Hodge et al. (2018) is shown by a black line and the envelope of manual DEM results in this study is shown by the grey polygon. 

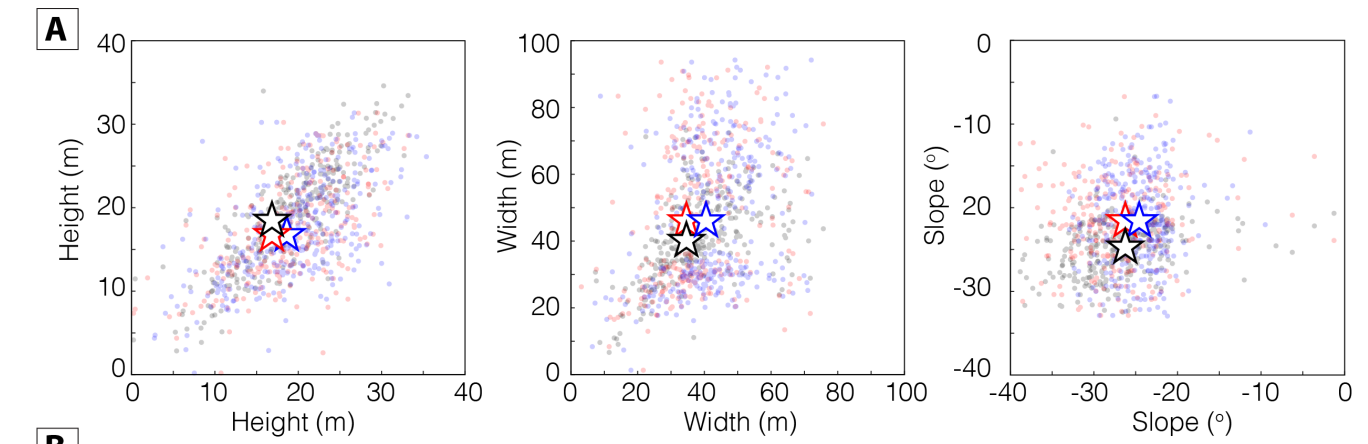

Pleaides $\vee$ TanDEM-X

Pleaides $\vee$ SRTM

- TanDEM-X v SRTM

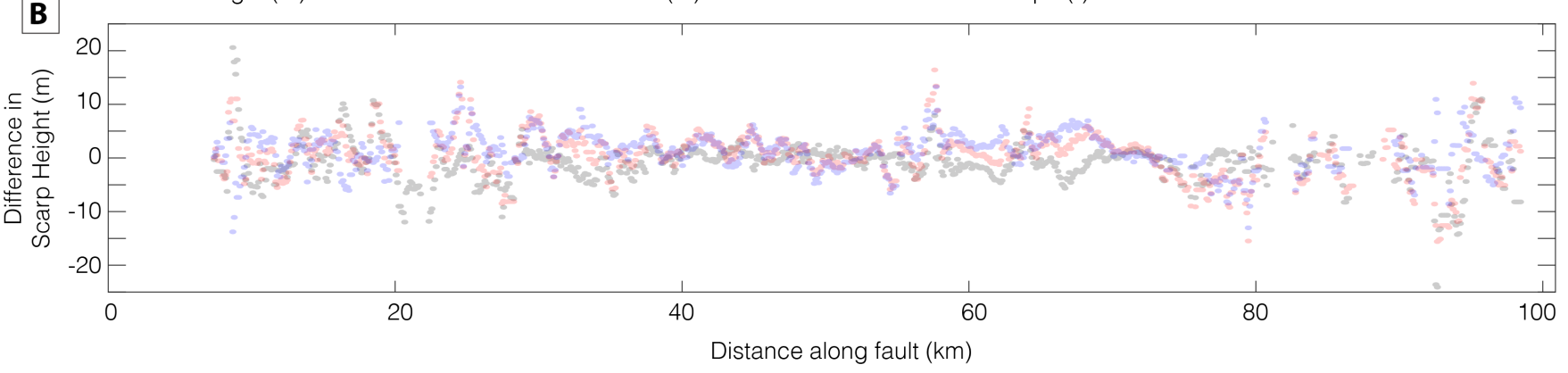

Figure S12. a) A comparison of algorithm scarp parameters for all DEMs (Pleiades, TanDEM-X and SRTM). b) The along-strike error between scarp height measurements for each DEM using the algorithm. 


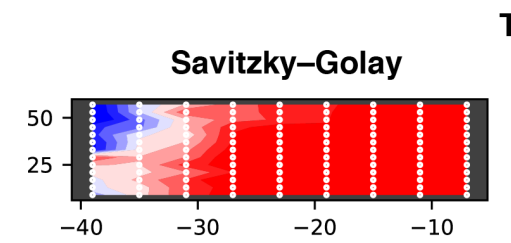

TOF
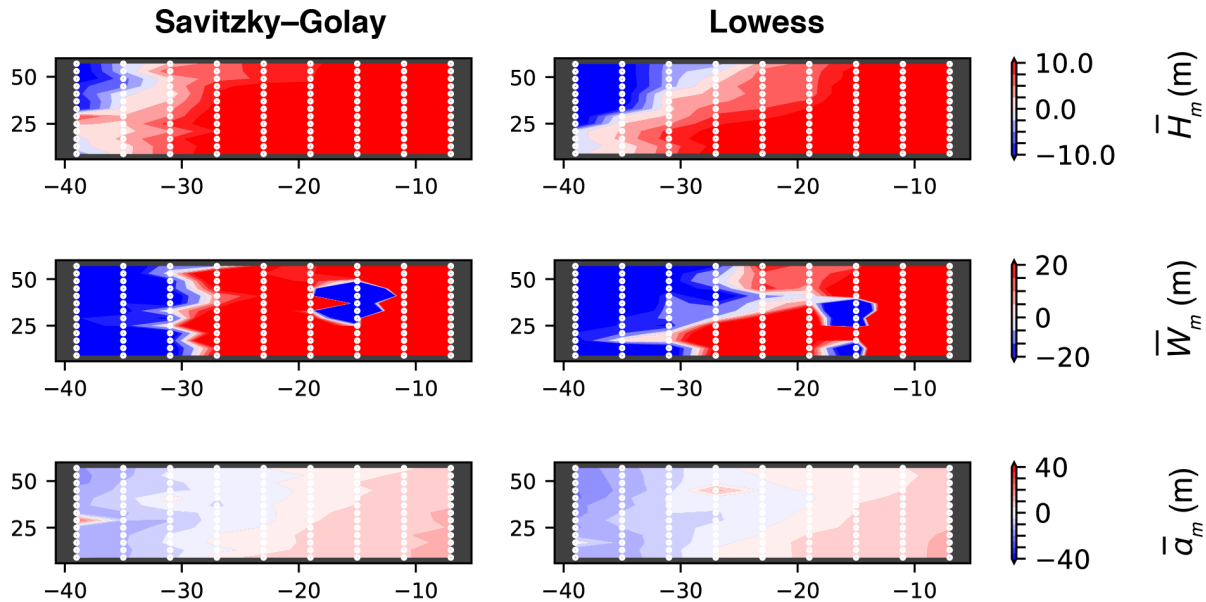

$\mathbb{E}_{-40}^{40} \quad 10^{E}$
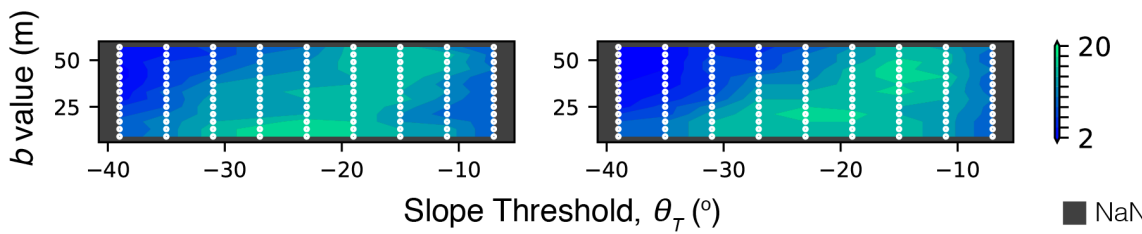

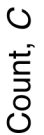

Slope Threshold, $\theta_{T}\left(^{\circ}\right)$

$\mathrm{NaN}$
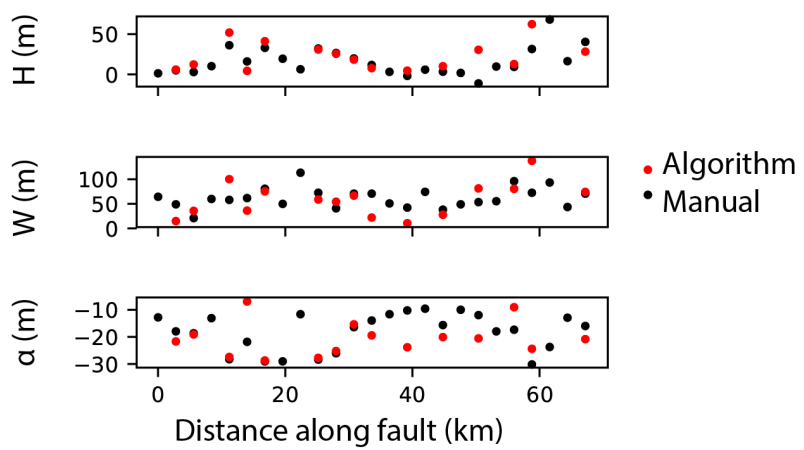

Figure S13. Top: The misfit analysis for the Thyolo fault (TOF). Bottom: The misfit between manual analysis and algorithm using the best performing parameters for twenty-five selected profiles. 


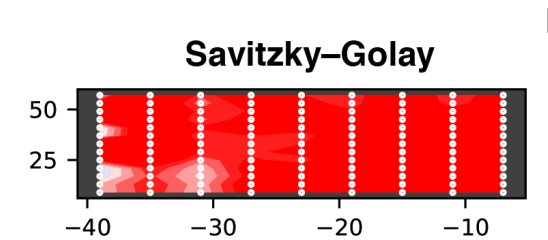

MOF
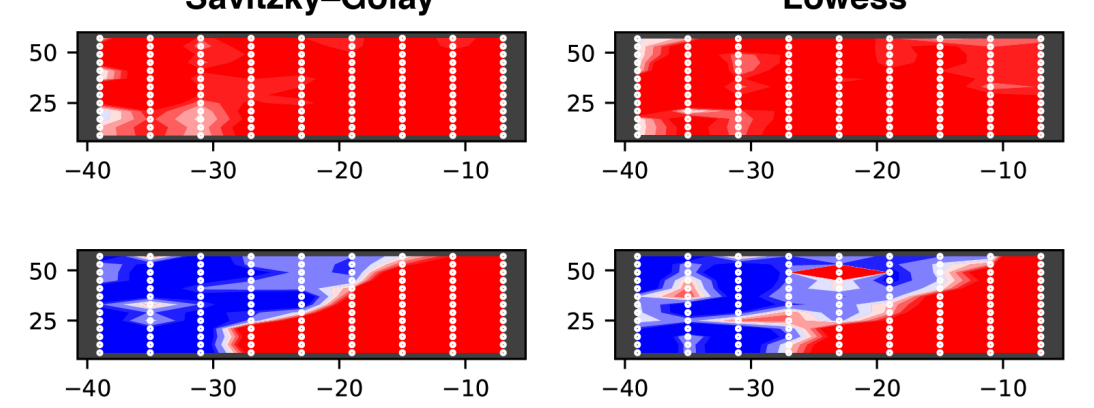

$\mathbb{E}_{-10.0}^{10.0} \widehat{\underline{\Xi}}$
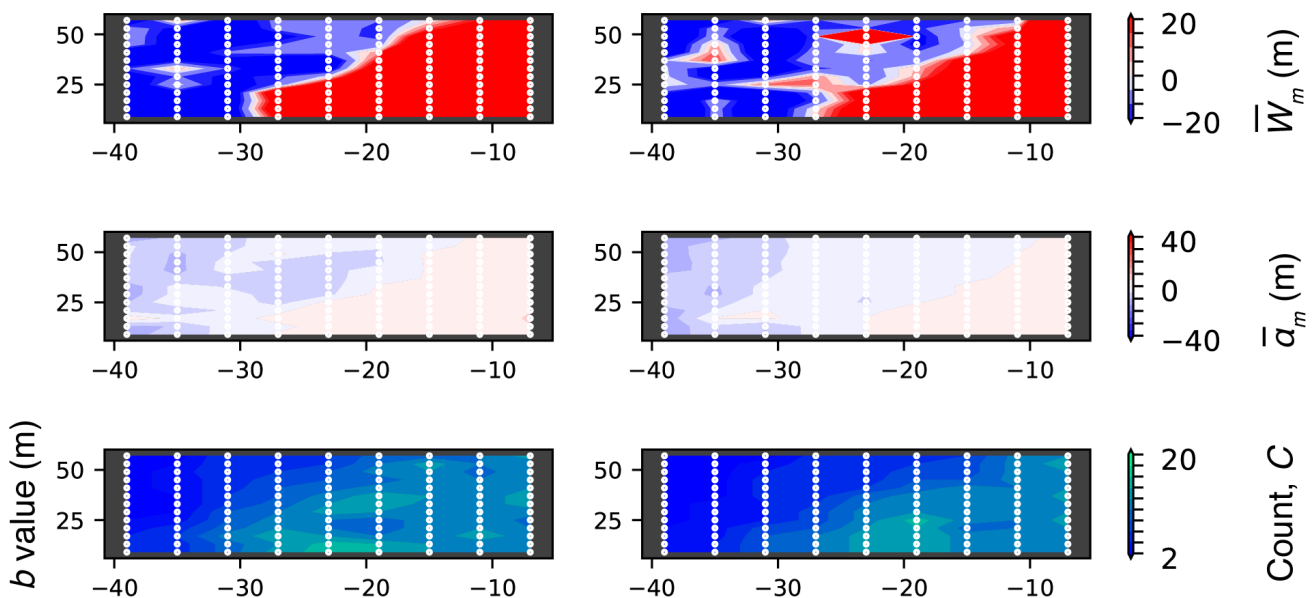

Slope Threshold, $\theta_{T}\left({ }^{\circ}\right)$

$\mathrm{NaN}$
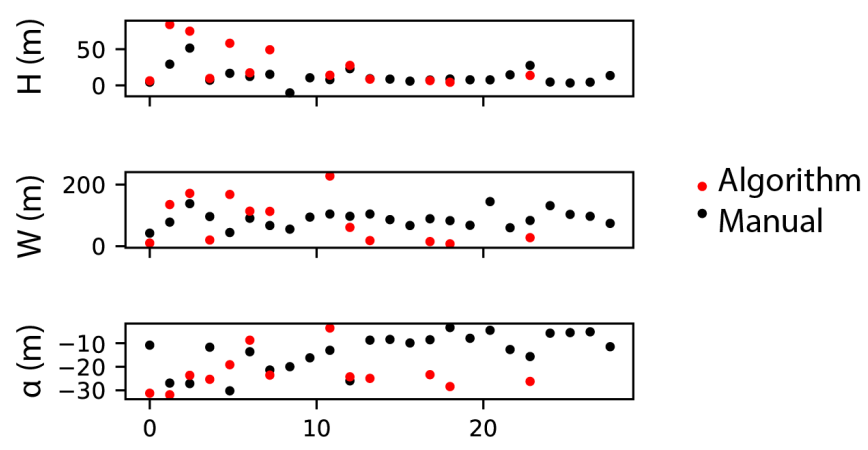

Distance along fault $(\mathrm{km})$

Figure S14. Top: The misfit analysis for the Muona fault (MOF). Bottom: The misfit between manual analysis and algorithm using the best performing parameters for twenty-five selected profiles. 


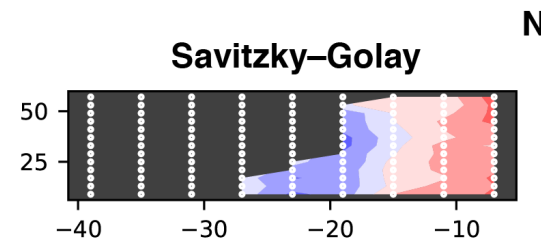

NMAF

Lowess
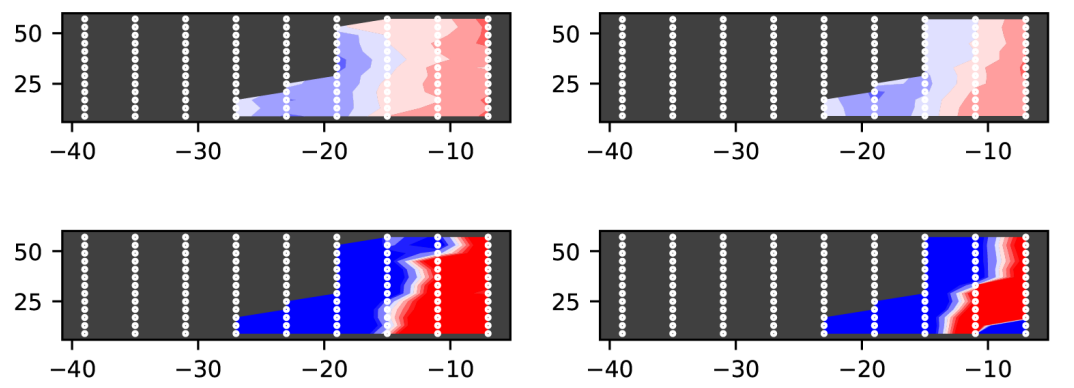

$\mathbb{E}_{-10.0}^{E_{0.0}^{10.0}} \hat{\underline{E}}$

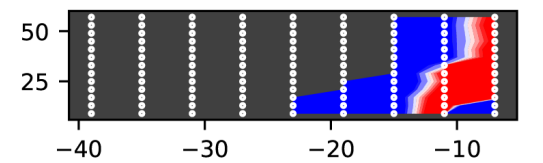

$\mathbb{E}_{-20}^{E_{0}^{20}} \overline{\mathbb{E}^{E}}$
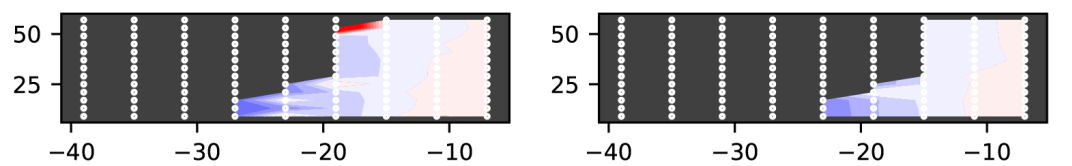

$\mathbb{E}_{-40}^{E_{0}^{40}} \frac{\widehat{E}}{10^{E}}$
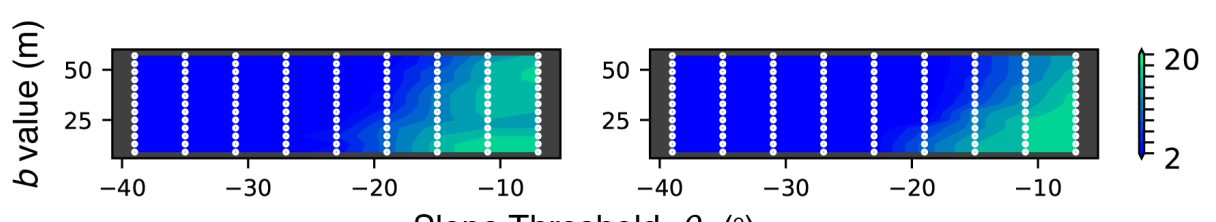

\begin{tabular}{l}
0 \\
\multirow{2}{*}{}
\end{tabular}

Slope Threshold, $\theta_{T}\left(^{\circ}\right)$

$\mathrm{NaN}$
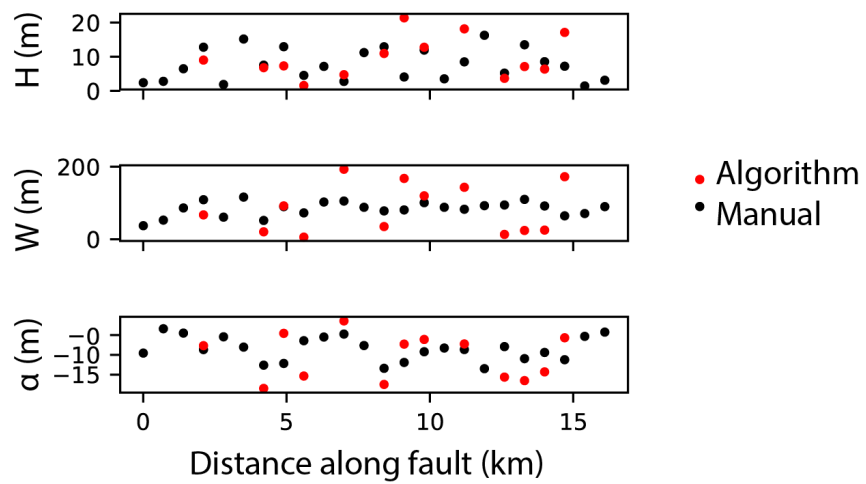

Figure S15. Top: The misfit analysis for the northern Malombe fault (NMAF). Bottom: The misfit between manual analysis and algorithm using the best performing parameters for twenty-five selected profiles. 

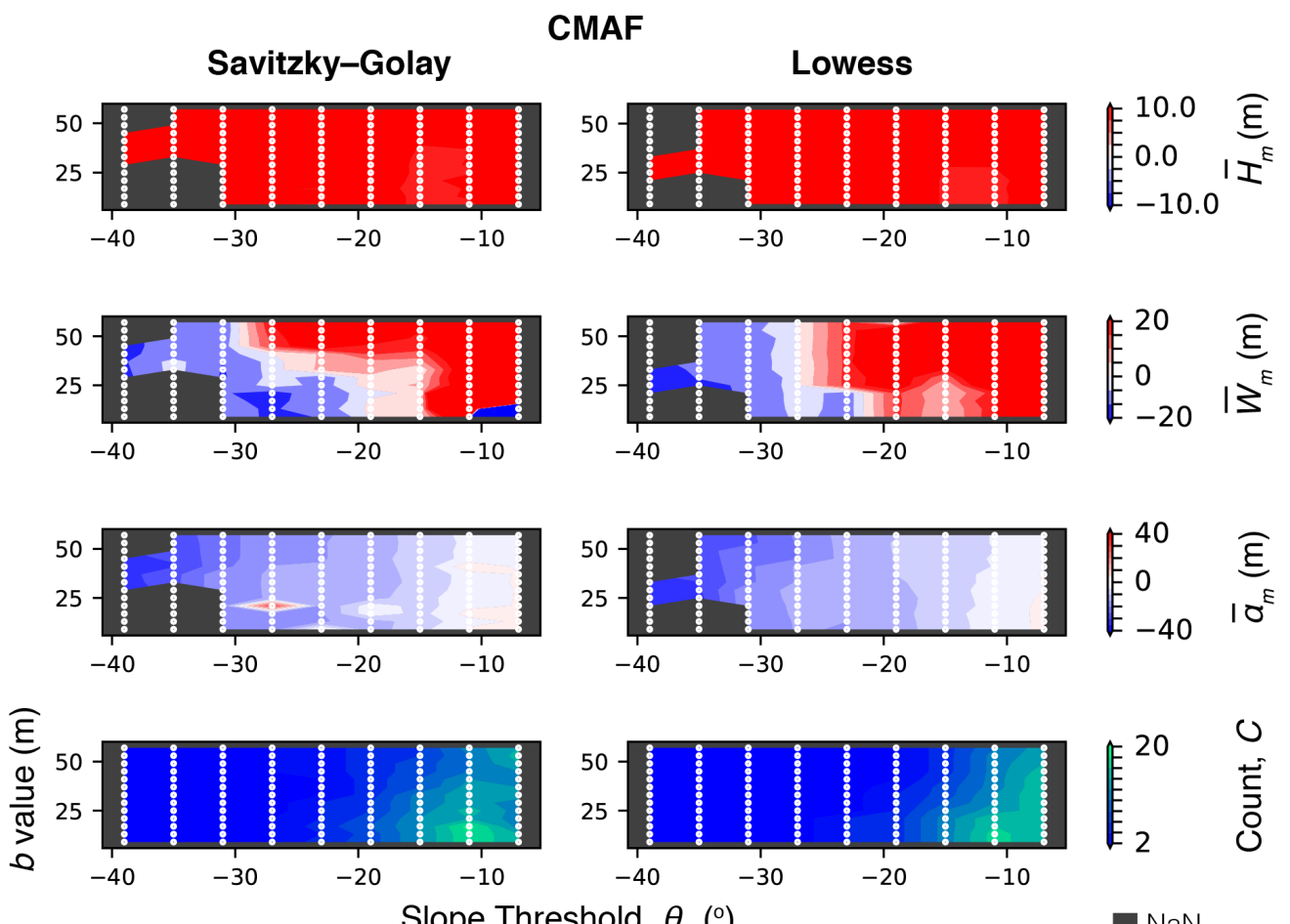

Slope Threshold, $\theta_{T}\left({ }^{\circ}\right)$

NaN
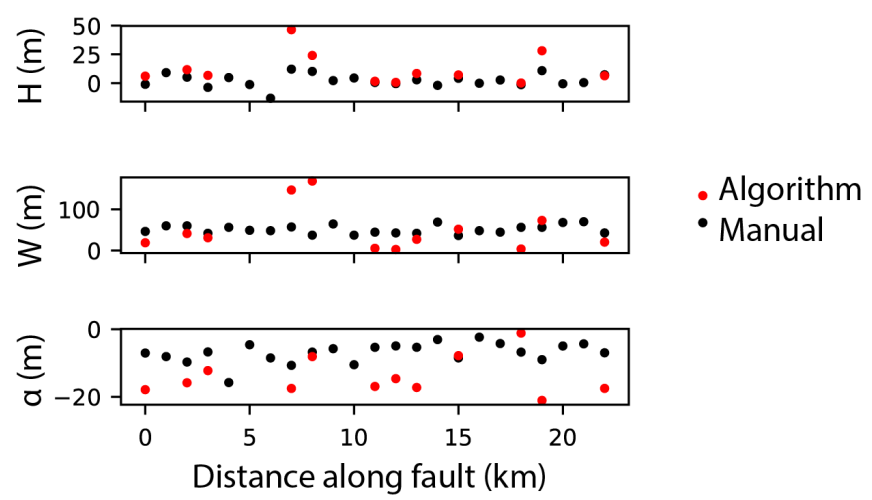

Figure S16. Top: The misfit analysis for the central Malombe fault (CMAF). Bottom: The misfit between manual analysis and algorithm using the best performing parameters for twenty-five selected profiles. 


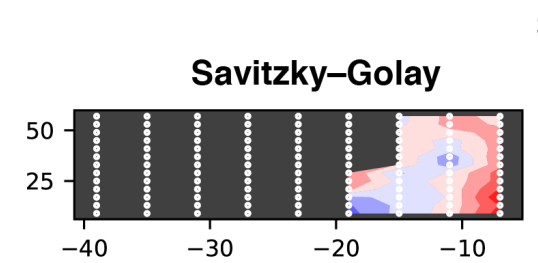

SMAF

Lowess
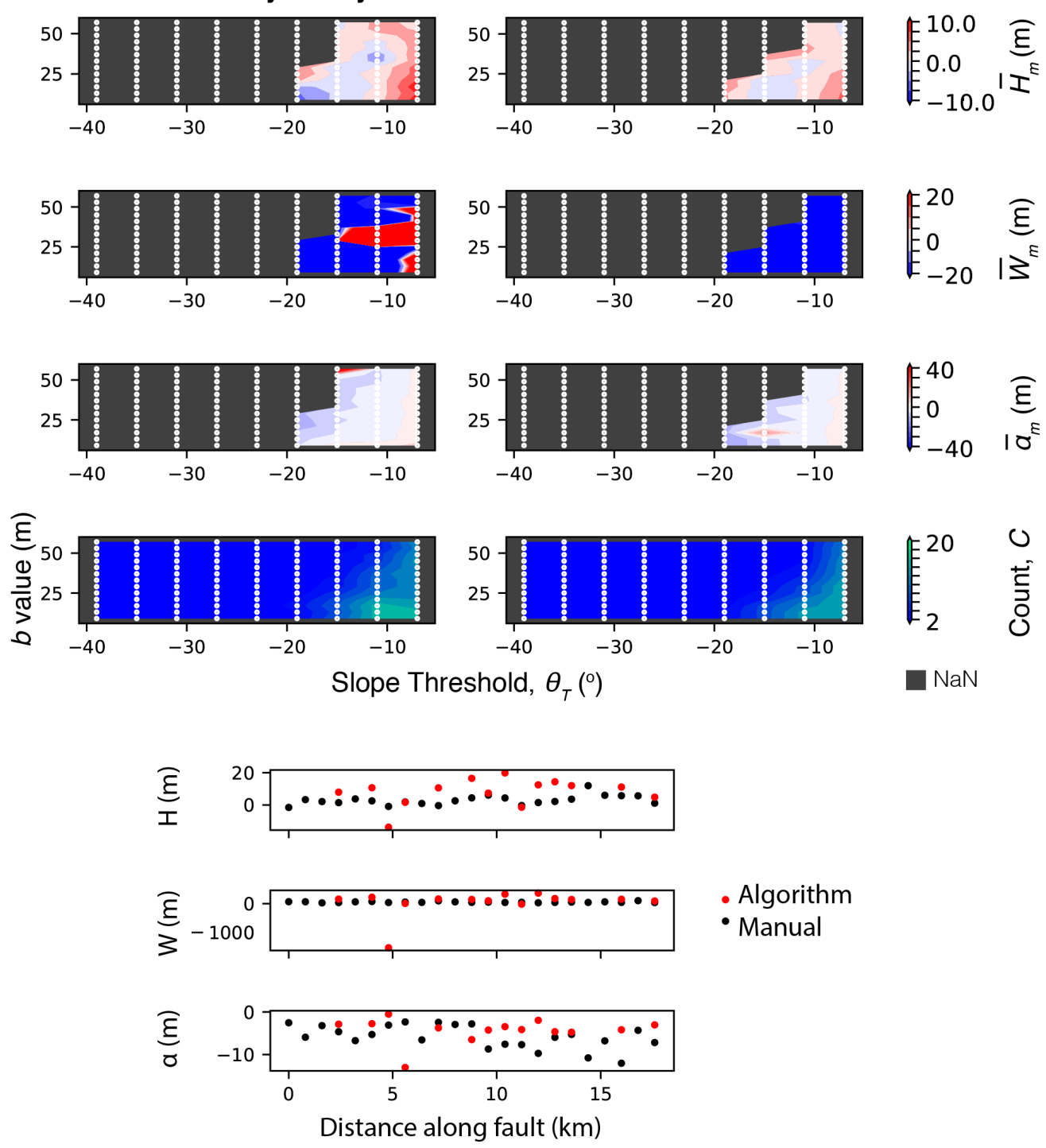

Figure S17. Top: The misfit analysis for the southern Malombe fault (SMAF). Bottom: The misfit between manual analysis and algorithm using the best performing parameters for twenty-five selected profiles. 
Table S1. A description and the parameters used to create five noise-free synthetic catalogue fault scarp profiles of varying morphology.

\begin{tabular}{llccccc}
\hline & Description & $H(\mathrm{~m})$ & $W(\mathrm{~m})$ & $\alpha\left(^{\circ}\right)$ & $\beta_{u}\left(^{\circ}\right)$ & $\beta_{l}\left(^{\circ}\right)$ \\
\hline 1 & Randomly selected profile & 25 & 37 & 35 & 1 & 2 \\
2 & Small scarp height & 4 & 9 & 29 & 4 & 4 \\
3 & Steep, large scarp & 39 & 10 & 75 & 3 & 5 \\
4 & Gentle original surfaces & 25 & 14 & 20 & 1 & 1 \\
5 & Variable original surfaces & 27 & 33 & 41 & 5 & 1 \\
\hline
\end{tabular}

Table S2. A description and the parameters used to create three noisy synthetic catalogue fault scarp profiles of varying amounts of noise and diffusion.

\begin{tabular}{llccccc}
\hline & Signal-to-noise ratio & Veg N $^{o}$ & Hill N $^{o}$ & Ditch N $^{o}$ & $t(\mathrm{kyr})$ & $\kappa\left(\mathrm{m}^{2} / k y r\right)$ \\
\hline 6 & High & 17 & 0 & 0 & 0 & 0 \\
7 & Medium & 0 & 1 & 2 & 16 & 2.3 \\
8 & Low & 15 & 2 & 2 & 5 & 4.5 \\
\hline
\end{tabular}


Table S3. Manually derived scarp parameters for twenty Bilila-Mtakataka fault scarp profiles using the $5 \mathrm{~m}$ Pleiades, $12 \mathrm{~m}$ TanDEM-X and 30 m SRTM DEMs.

\begin{tabular}{|c|c|c|c|c|c|c|c|c|c|c|}
\hline & \multirow[b]{2}{*}{ Dist (km) } & \multicolumn{3}{|c|}{ Pleiades } & \multicolumn{3}{|c|}{ TanDEM-X } & \multicolumn{3}{|c|}{ SRTM } \\
\hline & & $\mathrm{H}(\mathrm{m})$ & W (m) & $\alpha\left(^{o}\right)$ & $\mathrm{H}(\mathrm{m})$ & W (m) & $\alpha\left(^{o}\right)$ & $\mathrm{H}(\mathrm{m})$ & $\mathrm{W}(\mathrm{m})$ & $\alpha\left(^{o}\right)$ \\
\hline 1 & 10.3 & 19 & 30 & -32 & 21 & 30 & -35 & 9 & 40 & -13 \\
\hline 2 & 14.9 & 14 & 50 & -16 & 14 & 50 & -16 & 15 & 30 & -27 \\
\hline 3 & 19.5 & 26 & 30 & -41 & 15 & 50 & -17 & 15 & 50 & -17 \\
\hline 4 & 24.1 & 20 & 50 & -22 & 18 & 30 & -31 & 19 & 50 & -21 \\
\hline 5 & 28.7 & 26 & 50 & -27 & 20 & 50 & -22 & 12 & 40 & -17 \\
\hline 6 & 33.3 & 14 & 50 & -16 & 17 & 50 & -19 & 18 & 50 & -20 \\
\hline 7 & 37.9 & 22 & 40 & -29 & 21 & 40 & -28 & 14 & 50 & -16 \\
\hline 8 & 42.5 & 25 & 40 & -32 & 27 & 50 & -28 & 25 & 50 & -27 \\
\hline 9 & 47.1 & 24 & 50 & -26 & 26 & 50 & -27 & 22 & 40 & -29 \\
\hline 10 & 51.7 & 19 & 40 & -25 & 21 & 50 & -23 & 18 & 40 & -24 \\
\hline 11 & 56.3 & 13 & 20 & -33 & 23 & 50 & -25 & 31 & 80 & -21 \\
\hline 12 & 60.9 & 31 & 40 & -38 & 25 & 40 & -32 & 31 & 60 & -27 \\
\hline 13 & 65.5 & 28 & 30 & -43 & 30 & 70 & -23 & 24 & 60 & -22 \\
\hline 14 & 70.1 & 21 & 30 & -35 & 19 & 30 & -32 & 21 & 70 & -17 \\
\hline 15 & 74.7 & 14 & 30 & -25 & 14 & 20 & -35 & 21 & 60 & -19 \\
\hline 16 & 79.3 & 13 & 20 & -33 & 15 & 20 & -37 & 11 & 20 & -29 \\
\hline 17 & 83.9 & 8 & 10 & -39 & 7 & 20 & -19 & 6 & 10 & -31 \\
\hline 18 & 88.5 & 5 & 10 & -27 & 8 & 30 & -15 & 7 & 10 & -35 \\
\hline 19 & 93.1 & 3 & 10 & -17 & - & - & - & 7 & 10 & -35 \\
\hline 20 & 97.7 & 8 & 20 & -22 & 20 & 60 & -18 & 11 & 40 & -15 \\
\hline
\end{tabular}

Table S4. Average misfit values for the Bilila-Mtakataka fault using all DEMs for the best performing algorithm parameters using the Pleiades $5 \mathrm{~m}$ DEM (Lowess filter, bin width of $39 \mathrm{~m}$, slope threshold of $-21^{\circ}$ and slope derivative threshold of $5^{\circ} / \mathrm{m}$ ).

\begin{tabular}{lcccccc}
\hline & \multicolumn{3}{c}{ Synthetic } & \multicolumn{3}{c}{ Bilila-Mtakataka } \\
Value & 5 & 10 & 30 & Pleiades & TanDEM-X & SRTM \\
\hline Height $\bar{H}_{m}(\mathrm{~m})$ & -2.7 & -2.4 & -2.2 & 1.4 & -0.2 & -3.5 \\
Width $\bar{W}_{m}(\mathrm{~m})$ & 3.6 & 3.3 & 7.1 & -6.6 & -8.4 & 3.7 \\
Slope $\bar{\alpha}_{m}\left(^{\circ}\right)$ & -12.2 & -12.5 & -12.6 & 3.7 & 2.3 & -1.6 \\
\hline
\end{tabular}


Table S5. Algorithm scarp parameters for the Bilila-Mtakataka fault. Scarp height, width and slope are given as averages with an error of one standard deviation error.

\begin{tabular}{lccc}
\hline Value & Pleiades & TanDEM-X & SRTM \\
\hline Count, $C$ & 719 & 610 & 581 \\
Height (m) & $19 \pm 17$ & $21 \pm 11$ & $21 \pm 13$ \\
Width (m) & $73 \pm 71$ & $46 \pm 48$ & $61 \pm 63$ \\
Slope $\left(^{\circ}\right)$ & $20 \pm 12$ & $23 \pm 9$ & $21 \pm 6$ \\
\hline
\end{tabular}

Table S6. The $2 \sigma$ maximum and minimum values used in the algorithm quality check for the Bilila-Mtakataka fault.

\begin{tabular}{lccc}
\hline Value & Pleiades & TanDEM-X & SRTM \\
\hline Max Height (m) & 37 & 35 & 33 \\
Max Width (m) & 77 & 71 & 88 \\
Max Slope $\left(^{\circ}\right)$ & 44 & 40 & 33 \\
\hline
\end{tabular}

Table S7. Quality checked ( $2 \sigma$ of original data) algorithm scarp parameters for the Bilila-Mtakataka fault. Scarp height, width and slope are given as averages with an error of one standard deviation error.

\begin{tabular}{lccc}
\hline Value & Pleiades & TanDEM-X & SRTM \\
\hline Count, $C$ & 496 & 546 & 489 \\
Height (m) & $16 \pm 9$ & $17 \pm 9$ & $16 \pm 8$ \\
Width (m) & $26 \pm 18$ & $26 \pm 17$ & $23 \pm 24$ \\
Slope $\left(^{\circ}\right)$ & $23 \pm 10$ & $22 \pm 8$ & $19 \pm 8$ \\
\hline
\end{tabular}

